\title{
DIFFERENT POWER LOADING ALLOCATION SCHEMES FOR OFDM BASED COGNITIVE RADIO SYSTEM
}

Ajitsinh. N. Jadhav ${ }^{1}$ and Sakib. R. Mujawar ${ }^{2}$

\begin{abstract}
In advance wireless technology, security and scarcity of the spectrum are serious problem. With increase in advance wireless technology and demand for wireless multimedia services, facing lack of spectrum. As the radio frequency spectrum is a scarce natural resource so its efficient use is very important. A potential solution to this issue is to allocate the spectrum dynamically by means of cognitive radio system. In this paper, various powers loading algorithm like uniform, water-filling, sub-optimal and optimal algorithm for an OFDM base CR system has been investigated in simulation environment. Present numerical result show that optimal and sub optimal allocation algorithm can achieve higher transmission compare to classical power allocation algorithms namely, uniform and water filling power allocation algorithm. The above power loading algorithm is compared with respective to total transmission power, interference and individual peak power constraints.

Keywords - Cognitive Radio, Orthogonal Frequency Division Multiplexing, Primary User, Secondary User.
\end{abstract}

\section{I.NTRODUCTION}

Now a day we are facing many problems regarding spectrums due to the growing application in wireless fields. Lot of wireless applications is sharing the same medium and due to this there is overburden which leads to shortage of spectrum in given frequency bands. On the other hand, measurement show that many spectrum bands are rarely used most of the time, whereas other bands are used heavily crowded and as we know the radio frequency is limited natural resource and its efficient use is of the almost very importance. Moreover, most spectrum bands are allocated to certain services but spectrum occupancy measurements show that only some portions of the spectrum band are heavily used. The use of radio spectrum can be improved by means of the cognitive radio (CR) technology. In some case, the spectrum bands are not been efficiently utilized because licensed user are absent and cannot occupy their spectrum and unlicensed users are not allowed to operate in same spectrum bands.

Spectral efficiency can be improved significantly by giving Opportunistic access of the frequency bands to a group of required users (secondary CR users) for whom the band has not

\footnotetext{
${ }^{1}$ Department of Electronics \& Telecommunication D. Y. Patil college of Engineering \& Technology Kolhapur

${ }^{2}$ Department of Electronics \& Telecommunication D. Y. Patil college of Engineering \& Technology Kolhapur
} 
been licensed. Cognitive radio (CR) has been proposed as a way to increased spectrum efficiency by using the unused spectrum in dynamically changing environmental technology.

A Cognitive radio (CR) network is a technology in which a frequency band used by one or multiple primary users in a primary network can be operated by a secondary user's network which consists of one or multiple secondary users. To guaranty the quality of service (QoS) of the Primary User (PU) and to Maximize the transmission rate of the secondary users is one of the most crucial role for a Cognitive radio system. Orthogonal frequency division multiplexing (OFDM) is a promising tech for cognitive radio systems. With OFDM, the SU has the ability to smoothly flexible fill the spectral gaps left unused by PUs. It also can determine its location; sense spectrums of other devices, and change in frequency, adjust output power and even alter transmission parameters and characteristics. It can fulfil the SUs communication needs without altering the FCC rules. Cognitive radio mainly works on three tasks including: Radio scene analysis in which CR can detect spectrum holes, lightly used band, interference and Channel state estimation, in which CR determine the channel capacity and the state of the channel; Spectrum management, in which CR make the spectrum sharing efficient. The CR design is an featuring new methods of radio design philosophy which involves smoothly sensing the vacant spectrum and then determining the transmission characteristics (e.g., symbol rate, power, bandwidth, latency) of a group of secondary users based on the behaviour of the users to whom the spectrum has been licensed (referred to as primary users). Although opportunistic spectrum access would allow CR user to discover and access available spectrum resources, one of the main aim is to utilize the available spectrum resources in an efficient manner. OFDM base CR cognitive Radio:-OFDM is a multi-carrier modulation technique that can overcome many problems that arise with high bit-rate communications, the most serious of which is time dispersion. The data containing symbol stream is split into several low rate streams, and these streams are transmitted on different carriers. Because this splitting increases the symbol duration by orthogonally overlapping carriers (subcarriers), multipath echoes affect only a small part of the neighbouring symbols. The remaining inter-symbol interference (ISI) is removed by stretching of the OFDM symbol with a cyclic prefix (CP). Other advantages of OFDM include high spectral efficiency and robustness against narrowband interference (NBI).

Orthogonal division multiplexing (OFDM), because of its smooth flexibility in allocating the spectrum, has been recognized as a best air interface technology for CR systems. Because of the coexistence of $\mathrm{CR}$ and primary users is near to each other of bands, mutual interference between these users is the limiting factor in order to achieve a better performance for CR systems. Use of the classical power allocation Algorithms like water-filling algorithm for CR systems may result in higher interference to the primary user (PU) receivers. In, we propose a power loading algorithm that maximized the downlink transmission rate while keeping the total interference introduced to different PU receivers below a threshold level .A dispense algorithm for optimal resource allocation in OFDMA based CR systems has been proposed. When using orthogonal frequency Division Multiplexing in cognitive radio network, the power allocation schemes for spectrum resources will be convenient and very flexible. However, it become challenging Role to allocate power to individual sub channels in the OFDM-based cognitive radio networks. Due to the above reasons, OFDM-based CR systems have more attention and the related resource allocation problems have become good research topics.

\section{SYSTEM MODEL}


In the frequency domain, we consider that a CR user and L Pus co-exist in side by side bands. We consider a wireless system consisting of $M$ sub channels licensed to different primary users. Each of this PUs behaves differently in their band. All the sub-channels are divided into multiple subcarriers and they are opportunistically available to some secondary or cognitive user which uses the band in OFDM fashion we assume that total number of subcarriers are N. Fig. 1 shows such a system with $\mathrm{M}$ sub-channels licensed to different primary users and are divided into multiple subcarriers.

\subsection{Underlay Model}

We consider downlink transmission conduction. It is assumed that the frequency bands of bandwidth F1, F2 ... FL have been occupied by PU1, PU2... PUL. As in Figure 2.1, SUs can occupy either the spectrum of PUs or the side spectrum of PUs. The available bandwidth for CR transmission is divided into $\mathrm{N}$ subcarriers based OFDM system, and the bandwidth for each subcarrier is $\Delta f H z$.

In the downlink transmission conduction, there are three instantaneous fading gains: between the SUs transmitter and SUs receiver for the $i$ th subcarrier denoted as $h_{i}^{g s}$; between the SUs transmitter and $l$ th PU receiver denoted as $h_{l}^{s p}$; between $l$ th Pus transmitter and SUs receiver denoted as $h_{i}^{p z}$. We assume that these instantaneous fading gains are perfectly known at the SUs transmitter.

\subsubsection{Interference introduced to $\mathrm{PU}$ by $\mathrm{SU}$}

We assume that the signal transmitted on the subcarrier is an ideal. The power spectrum density of the $i$ th subcarrier can be written as:
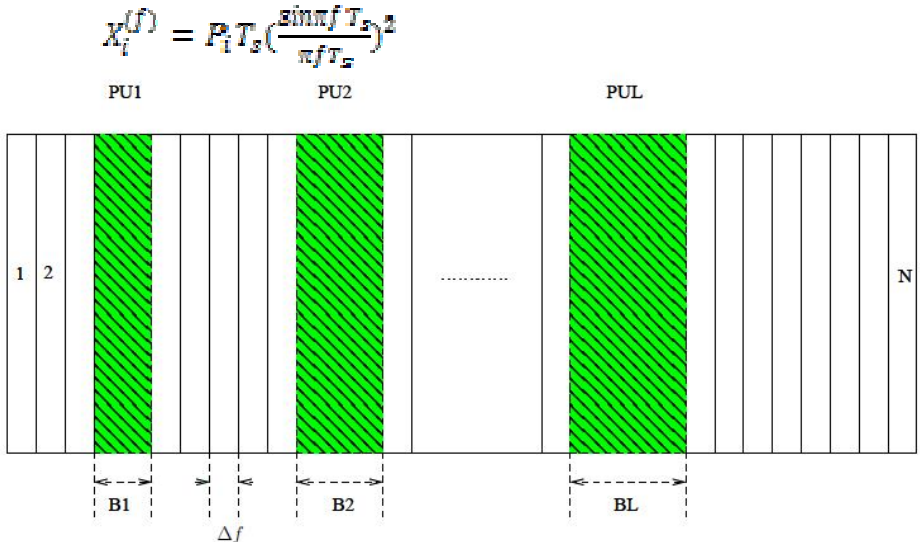

Fig. 2.1 Illustration for underlay Model

Where $P_{i}$ is the total transmits power in the $i$ th subcarrier and $T_{s}$ is the symbol duration.

Then the interference introduced to the $l$ th PU band by the $i$ th subcarrier is:

$$
l_{i}^{00}=P_{I} \mid h_{l}^{s p} \|^{2} T_{g} \int_{d_{i L}-\frac{\Xi_{i}}{2}}^{d_{i L}+\frac{\Xi_{i}}{2}}\left(\frac{\pi n m f T_{s}}{\pi f T_{s}}\right)^{2} d f
$$

Where $d_{i l} i s$ the distance in frequency between the $i$ th subcarrier and the $l$ th PU band, and $B_{l}$ represents occupied bandwidth by the $l$ th PU. 


\subsubsection{Interference introduced to SU by PU}

The power spectrum density of the PU signal after M-FFT processing can be expressed as:

$$
E\left[I_{N}(\omega)\right]=\frac{1}{2 \pi M} \int_{-\pi}^{\pi} X_{P V}\left(e^{j \omega)}\left(\frac{\sin \left(\omega-v^{2} M\right.}{2}\right)^{2} d y\right.
$$

Where $X_{P U}\left(\mathrm{e}^{j \omega}\right)$ is the power spectrum density of the PU signal. The PU signal has been taken to be an filtered white noise process with amplitude $\mathrm{P}_{\mathrm{PU}}$. According to the interference introduced to the $i$ th subcarrier by the $l$ th PU band can be written as:

$$
J_{i}^{\omega}\left(P_{P V}\right)=\left|h_{I}^{s p}\right|^{2} \int_{d_{i L}-\frac{\Delta_{f}}{2}}^{d_{i L}+\frac{\Delta_{f}}{2}} E\left[I_{M}(\omega)\right] d \omega
$$

\section{ProblemFormulation And Optimal Power Allocation}

The design goal is to find power value for each subcarrier, $\mathrm{P} i(i=1,2 \ldots \mathrm{N})$ For given instantaneous fading gain $h_{i}^{g s}$, given fading statistics of $h_{l}^{s p}$ and the total transmit power budget $\mathrm{P}_{T}$. As such the total transmission rate of the $\mathrm{CR}$ user, $\mathrm{C}$ is maximized while the probability that the interference introduced to 1 th $(l=1,2 \ldots \mathrm{L})$ PU band is kept below the threshold $I_{\text {th }}^{l}(1=1,2 \ldots$ L), respectively, with the probability value $\alpha$ or above. Mathematically, the problem can be formulated as a constrained optimization problem as follows.

$$
C=\sum_{p i=1}^{\max } \log _{2}\left(1+\frac{\left|h_{i}^{2}\right|^{2} p_{i}}{\sigma^{2}+\sum_{i=1}^{2} J_{i}^{0}}\right)
$$

Subject to:

$$
\begin{gathered}
P_{\mathrm{r}}\left(\sum_{i=1}^{N} I_{i}^{0}\left(a_{\mathrm{i}}, P_{\mathrm{i}}\right) \leq I_{z h}^{0}\right) \geq a, \quad \forall, \\
P_{1} \geq 0, \quad \forall h \\
\sum_{i=1}^{N} P_{i} \leq P_{T}
\end{gathered}
$$

Where $\mathrm{P}_{\mathrm{r}}$. denotes the probability. Now the probabilistic interference constraint in Eq. (6) can be written as.

$$
P_{r}\left(\left|h_{l}^{s p}\right|^{2} \sum_{i=1}^{N} S_{i}^{b} P_{i} \leq I_{t}^{b}\right) \alpha_{i} \quad \forall
$$




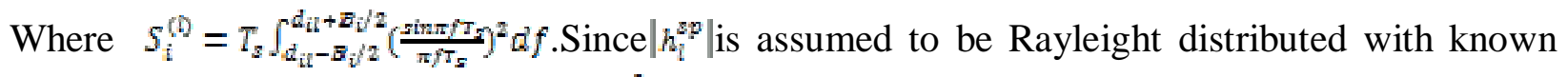
parameter $\lambda_{l}$, the distribution of $\left|h_{i}^{3 p}\right|^{2}$ corresponds to an exponential distribution with the parameter $\lambda$ i . The Eq. (9) can be evaluated in closed form for the Rayleigh fading case as follows

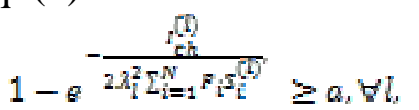

After some mathematical manipulations, Eq. (10) can be written as

$$
\sum_{i=1}^{N} P_{i} S^{D} \leq \frac{I_{t h}^{(D)}}{2 \lambda_{l}^{2}(-\ln (1-a))}, \quad \forall l
$$

The optimal power of the $i^{\text {th }}$ subcarrier is given by:

$$
B_{i}^{*}=\left[w_{i}-\frac{\sigma^{2}+\sum_{I=1}^{2} J_{i}^{(D)}}{\left|h_{i}^{s z}\right|^{2}}\right] \forall l
$$

Where $w_{i}=\frac{1}{\beta+\Sigma_{l=1}^{L} \gamma l k_{i}^{R}}$ and $\beta$ and $\gamma t$ are deterministic Lagrange parameters.

\section{IV.POWER LOADING SCHEMES}

In this section we describe classical algorithms namely uniform loading and water-filling algorithms which is used for conventional OFDM system and proposed optimal and sub optimal algorithm.

\section{A. Uniform Loading Algorithm}

In uniform power loading algorithm, which is used in the conventional OFDM systems due to its reduced complexity, equal amount of power is allocated in each subcarrier such that all $L$ +1 constraints in Eqs. (8) and (11) can be satisfied. By assuming equal power in each subcarrier and solving Eq. (11) to satisfy the equality on $i$ th interference constraint $I_{\text {in }}^{\text {the }}$ the corresponding power for $i$ th subcarrier can be written as

$$
P_{i}^{D}=\frac{l_{\mathrm{th}}^{\mathrm{D}}}{2 \sum_{i=1}^{N} 5_{i}^{(D)} \lambda_{l}^{2}(\ln 1 /(1-a))} \forall l
$$

The power allocation for constraint in Eq () can be written as

$$
P_{i}^{(l+1)}=P_{T} / N
$$

The final power allocation to each subcarrier is done according to

$$
P_{i}^{\operatorname{singst}}=\min \left\{P_{i}^{(1)}, P_{i}^{(2)}, \ldots, P_{1}^{(2)}, P_{i}^{(2+1)}\right\} \quad \forall i
$$


It is important that atlest one of these $\mathrm{L}+1$ constraints will be met and hence, scaling of power is not required.

\section{B. Water-filling Power Loading Algorithm}

In water-Filling scheme, which is optimal power allocation algorithm in conventional OFDM system, we use the total power allocated by uniform loading as the power constraint. The allocated power in the ith subcarrier because of the ith subcarrier because of the ith interference constraint is written as

$$
P_{i}^{D}=P / S_{i}^{D}, \quad \forall l_{i}
$$

By using Eq.(11), after nulling (2L-1) subcarriers $\mathrm{P}$ can calculated by assumption of strict uniformity in the equality in the $l$ th interference in Eq. (11). Using Eq. (13), this equality constraint can be written as

$$
\sum_{i=1}^{N} P_{i} S_{i}^{(D)}=\frac{I_{t h}^{(i)}}{2 \lambda_{i}^{2}(-\ln (1-a))}
$$

We can derive

$$
P=\frac{I_{t h}^{W}}{2(N-2 L+1) \lambda_{l}^{2}(-\ln (1-a))}
$$

Through Eq. 16 we can get $P_{i}^{\infty}$ as following

$$
P_{i}^{D}=\frac{I_{h}^{W}}{2 S_{i}^{\omega}(N-2 L+1) \lambda_{l}^{2}(-\ln (1-a))}
$$

Now we need to calculate power values $P^{[2+1]}$ due to the totalpower constraint. In order to meet the total power constraint, we use the standard water-filling algorithm to distribute total power PTamong $N$ CR subcarriers. According to the water-filling algorithm with a total power constraint $P T$, the power values can be written as

$$
P_{i}^{(L+1)}=\max \left(0, \frac{1}{\alpha}-\frac{\sigma^{2}+\Sigma_{i=1}^{L} J_{i}^{(D)}}{\left|h_{i}^{s s}\right|^{2}}\right) \forall i_{i}
$$

Where the Lagrange constant a can be calculated from the following eqution

$$
\sum_{i=1}^{N} \max \left(0, \frac{1}{\alpha}-\frac{\sigma^{2}+\sum_{i=1}^{L} j_{i}^{b}}{\left|h_{i}^{B}\right|^{2}}\right)=P_{T}
$$

The power value for $i$ th subcarrier, denoted by $P_{i}^{\left[W F_{3}\right.}$, are obtained using the standard waterfilling algorithms mentioned in Eq (20) and Eq (21) considering the total power constraint equalto the total power allocated by uniform loading algorithm. The power values will satisfy the total power constraint given in Eq. (11), however it is checked that if the power values satisfy the 
interference constraints specified in Eq. (8). If a particular interference constraint is not satisfied, the power value in each subcarrier $P$ is reduced such that the all interference constraints are satisfied. Also, if none of these interference constraints is met strictly, the power value increased until one of these interference constraints is met strictly.

\section{Sub-optimal Power Loading Algorithm}

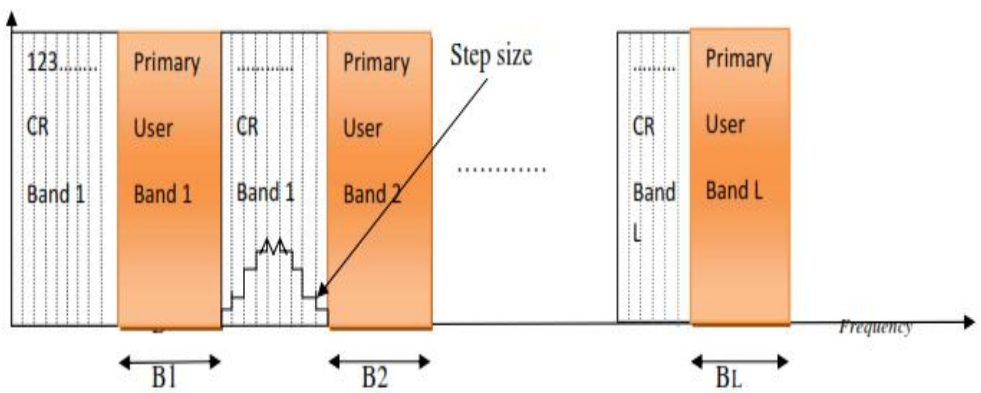

Fig.2 Ladder profile of power distribution

The power has to be assigned to $N$ CR subcarriers such that the transmission rate of CR user can be maximized while all $L+1$ (total power constraint from Eq. (8) and $L$ interference constraints from Eq. (11) constraints are to be satisfied. The complexity of the optimal algorithm comes from the fact that these $L+1$ constraint have to be met simultaneously. In order to reduce such complexity, we follow a two step procedure. First, we keep only one of the $L+1$ constraints and find power allocation sub-optimally in each subcarrier. Without loss of generality, let us denote that the allocated power in the $i$ th subcarrier due to the $l$ th constraint by $P_{i}^{0}$ Inorderto satisfy $l$ th interference constraint given in Eq.(11), power is allocated according to ladder profile as shown in figure 2. It is based on the heuristics that if a subcarrier is closer to a primary user band, it introduces more interference. Hence, less power should be allocated to that particular subcarrier. In particular, we propose to allocate power in each CR subcarrier such that the allocated power is inversely proportional to the factor $K_{i}^{p}$ that depends on the spectral distance between $i$ th $\mathrm{CR}$ subcarrier and the $l$ th PU band. Hence the power in the $i^{\text {th }}$ subcarrier can be written as

$$
F_{i}^{*}=P / S_{i}^{(1)}
$$

Where $\mathrm{P}$ will be determined by the value of Ith in following equation

$$
\sum_{i=1}^{N} S_{i} * P * i=I_{t h} \text {. }
$$

will hold in this case as well. By substituting Eq.(23) in Eq. (22) the power allocation policy can be expressed as. 


$$
P_{i}^{E}=\frac{I_{t h}}{N * S_{i}}
$$

\section{NUMERICAL RESULTS}

In this section we present simulation results where we assume that there are three PU bands $(L=3)$, and there are Five OFDM subcarriers $(N=5)$ for the CR user. The values of $T s, \Delta f, B 1$, $B 2$, and $B 3$ have been assigned, $0.3125 \mathrm{MHz}, 1 \mathrm{MHz}, 2 \mathrm{MHz}$, and $5 \mathrm{MHz}$, respectively. AWGN variance, $(\sigma 2)$ is assumed to be equal to $10^{-8} \mathrm{~W}$ and the channel fading gains are assumed to follow Rayleigh distribution. The average channel power gains for $\| h_{1}^{s z}||^{2},\left|h_{1}^{s p}\right|^{2},\left|h_{2}^{s p}\right|^{2}$ and $\left|h_{3}^{s y}\right|^{2}$ are assumed to be $-10 \mathrm{~dB},-5 \mathrm{~dB},-7 \mathrm{~B}$, and $-10 \mathrm{~dB}$, respectively. The values of $i_{\mathrm{i}}$ are generated randomly with an average value of $1 \times 10^{-6} \mathrm{~W}$. The values of $I_{t h}^{(1)}$, and $I_{t h}^{(2)}$ have been assumed to be $1 \times 10^{-6} \mathrm{~W}$, and $5 \times 10^{-6} \mathrm{~W}$, respectively.

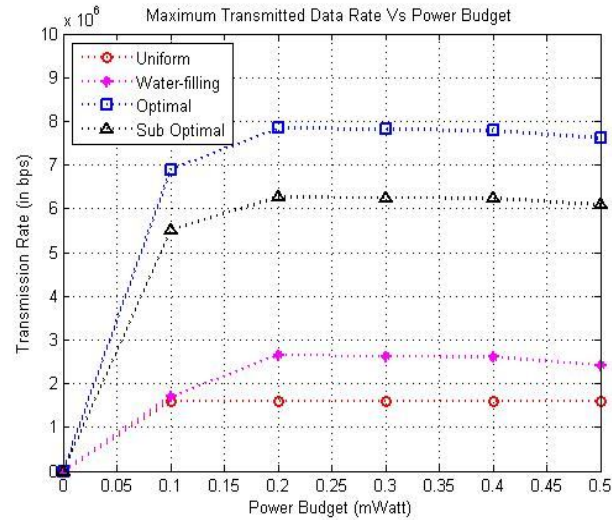

Fig.4 Transmission data rate for CR user vs interference threshold

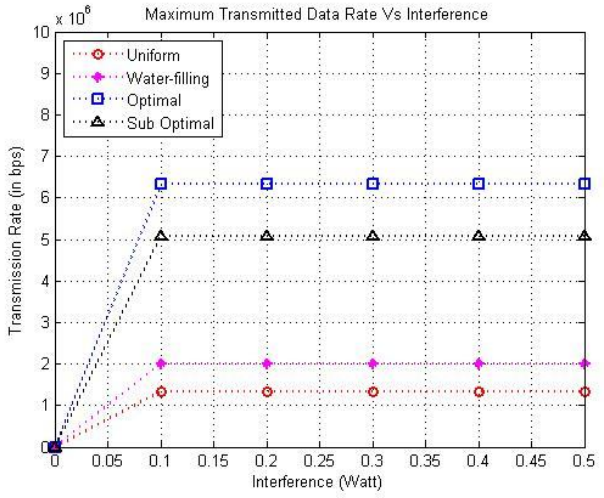

Fig.3 Maximum transmitted data rate vs power budget

In Figure.3, we plot the achievable maximum transmission rate for the CR user versus the total power budget for various algorithms. The value of $I_{t h}^{R}$ has been fixed to $2 \times 10^{-6} \mathrm{~W}$, the individual peak power constraint $G_{j}$ has been fixed to $2 \times 10^{-6} \mathrm{~W}$, and the value of $\alpha$ has been considered to be 0.95 . From this figure, we observe that the proposed optimal algorithm is able to achieve higher transmission rate for a given power budget than the suboptimal algorithm. It should be noted that as we increase the power budget for CR user, the interference constraint becomes dominant and the transmission rate of CR user does not increase as the power budget increases. This is expected as in this region the CR system operates in an interference limited scenario.In Figure.4, we plot the achievable transmission data rate for the CR user versus interference threshold for the second PU band, $\left(I_{t h}^{(2 \pi)}\right)$ for all the algorithms under consideration. The value of 
total transmit power, $P_{T}$ has been assumed to be $5 \times 10^{-4} \mathrm{~W}$, the individual peak power constraint $G j$ has been fixed to $2 \times 10^{-6} \mathrm{~W}$, and the value of $\alpha$ has been considered to be 0.95 . Again, we observe that the proposed. Optimal algorithm achieves higher transmission rate than that of other algorithms. The proposed suboptimal algorithm performs better than classical algorithms. Further, water- filling algorithm achieves higher transmission rate than the uniform algorithm. The transmission rate versus interference threshold curve saturates after a certain value of $\left(I_{\mathrm{th}}^{(2]}\right)$. The reason is that although is relaxed by increasing its value, other constraints $\left(\left(I_{\mathrm{th}}^{[1]}\right),\left(I_{\mathrm{th}}^{[3]}\right)\right.$ and $\mathrm{P}_{\mathrm{T}}$ ) becomes dominant.

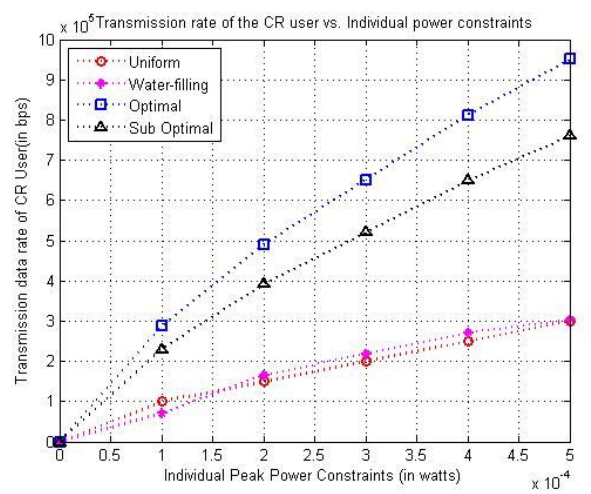

Fig.5 Transmission data rate for $\mathrm{CR}$ user vs Probability

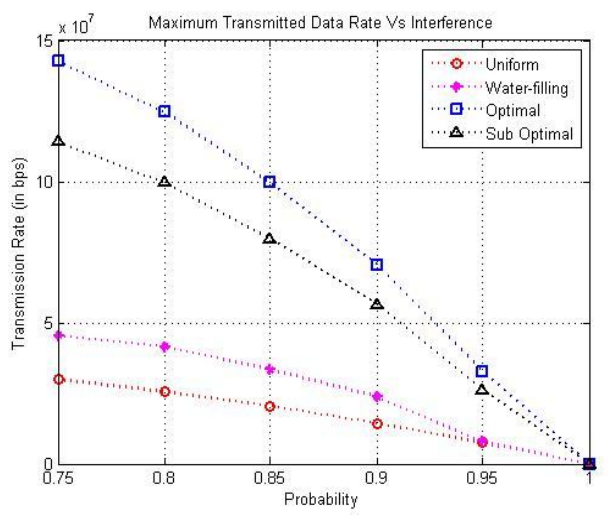

Fig.6 Transmission rate for the CR user versus individual power constraints

In Figure.5, we plot achievable transmission rate for the CR user versus probability $\alpha$. The individual peak power constraint $G j$ has been fixed to $2 \times 10^{-6} \mathrm{~W}$, the values of $P_{T}$, and $\left(I_{\mathrm{th}}^{(2 \pi)}\right.$ ), is assumed to be $5 \times 10^{-4} \mathrm{~W}$, and $2 \times 10^{-6} \mathrm{~W}$, respectively. As expected, we observe that the proposed optimal algorithm performs best over other algorithms. Also, suboptimal algorithm outperforms better than the water-filling algorithm, which performs better than the uniform power loading algorithm. It is observed from Figure 5.4 that as expected as the value of $\alpha$ increases, the achievable transmission rate of CR user decreases for a given power budget, individual peak power constraint, and interference thresholds.

In Figure.6, we plot the achievable maximum transmission rate for the $\mathrm{CR}$ user versus individual power constraints for various algorithms. The value of $I_{\text {th }}^{22}$ has been fixed to $2 \times 10-{ }^{6} W$, the total power $P_{T}$ has been fixed to be $5 \times 10^{-4} \mathrm{~W}$, and the value of $\alpha$ has been considered to be 0.95 . From this figure, we observe that the proposed optimal algorithm is able to achieve higher transmission rate for a given power budget than the suboptimal algorithm. It should be noted that as we increase the individual power constraint for CR user, the transmission rate of CR user does increase as the individual peak power constraint increases. This is expected as individual subcarrier can be allocated more power.

\section{CONCLUSION}

In this paper, first we develop a novel cognitive radio concept using underlay channel band and an optimal power allocation algorithm for the orthogonal frequency division multiplexing (OFDM)-based CR system with total power, interference and individual peak power constraints. Also we developed a suboptimal power allocation algorithm using nulling method for this problem in order to reduce the complexity of computation. As such the transmission rate of the 
CR user is maximized for a given total power budget, probabilistic interference and individual peak power constraints. Instead of instantaneous channel fading gains between the PU receivers and the CR transmitter, the developed power allocation algorithm requires the fading statistics and corresponding parameters to be known at the CR transmitter. Second, we propose and investigate performance of a low complexity suboptimal power allocation algorithm for subcarrier power allocation with total power and interference constraints. Simulation results have shown that our proposed optimal and suboptimal power allocation algorithms can achieve higher transmission rate for $\mathrm{CR}$ user compared to the existing power allocation algorithms namely, the uniform and water-filling power allocation algorithms. The proposed low complexity suboptimal algorithm achieves better performance than existing suboptimal, uniform and waterfilling power loading algorithms.

\section{REFERENCES}

[1]. Haykin, "Cognitive radio: brain-empowered wireless communications, IEEEJ.Sel .Areas Commun" vol.23,no.2,pp.201-220,Feb.2005

[2]. H.A.Mahmoud, T.Yucek, and H.Arslan,"OFDM for cognitive radio: Meritsandchallenges,"IEEE Wireless Commun.Mag.,vol.16,no.2, pp.6-15,Apr.2009

[3]. D. Ngo, C. Tellumbra, andH. H. Nguyen, "Resource allocation for OFDM-based cognitive radio multicast networks,"inProc.IEEE Wireless Commun. And Networking Conf.,pp.1-6,Apr.2009.

[4]. T. Weiss, J. Hillenbrand, A. Krohn, and F. K. Jondral, "Mutual interference in OFDM-based spectrum pooling systems," in Proc. IEEE Vehicular Technol. Conf. (VTC'04 Spring), vol. 4, pp. 1873-1877, May 2004.

[5]. Peter He, Lian Zhao, Sheng Zhou, "Water-Filling A geometric approach and its application to solve generalized radio resource allocation problem," in Proc. IEEE Transation on wireless communication. Conf, vol. 12,No.7 pp.1-10,July 2013.

[6]. P. Wang, M. Zhao, X. F. Zhong, L. M. Xiao, S. D. Zhou, and J. Wang, "Power Allocation in OFDM-Based Cognitive Radio Systems ,” IEEE Global Telecommunications Conference, pp. 4065-4065, 2007.

[7]. P. Kaligineedi, G. Bansal, and V. K. Bhargava, "Power Loading Algorithms for OFDM-Based Cognitive Radio Systems with Imperfect Sensing," IEEE Trans. Wireless Communications, vol. 11, pp. 4225-4230, Dec. 2012.

[8]. G. Bansal, P. Kaligineedi, and V. K. Bhargava, "Joint Sensing and Power Loading Algorithms for OFDMBased Cognitive Radio Systems," in Proc. IEEE Wireless Communications and Networking Conf., pp. 1-5, Apr. 2010.

[9]. G. Bansal, J. Hossain, and V. K. Bhargava, "Optimal and Suboptimal Power Allocation Schemes for OFDMbased Cognitive Radio Systems," IEEE Trans. Communications, vol. 7, pp. 4710 - 4718, Nov. 2008.

[10]. D. T. Ngo, C. Tellumbra, and H. H. Nguyen, "Resource allocation for OFDM-based cognitive radio multicast networks with primary user activity consideration," IEEE Transaction on Vehicular Technology, vol. 59, pp. 1668-1679, May 2010. 